programs to successfully prevent and control STDs may be complicated by recruitment of new sex partners at such events. Innovative collaborative prevention interventions at sex events and for residents returning from such events should be explored.

\section{P1-S5.09 ABSTRACT WITHDRAWN}

\section{P1-S5.10 ACUTE GENITAL ULCERATION IN SOUTH AFRICAN MEN: IMPORTANCE OF AGE AND SEXUAL RISK BEHAVIOURS}

\author{
doi:10.1136/sextrans-2011-050108.188
}

${ }^{1} \mathrm{~J}$ Leichliter, ${ }^{2} \mathrm{D}$ Lewis, ${ }^{3} \mathrm{G}$ Paz-Bailey. ${ }^{1}$ Centers for Disease Control \& Prevention, Atlanta, USA; ${ }^{2}$ NICD/NHLS, South Africa; ${ }^{3}$ Universidad del Valle de Guatemala, Guatemala

Background Herpes simplex virus (HSV) is the leading cause of genital ulcer disease (GUD) in Africa. Although some studies have examined the demographics and sexual behaviours of men with GUD, less is known about the differences that may exist between men with new sexually-acquired ulcers and men with other forms of GUD.

Methods Men with GUD ( $\mathrm{n}=615)$, who were 18 years and older, were recruited from several primary healthcare clinics in Gauteng Province, South Africa. Participants received syndromic management, were tested for ulcer aetiology and urethritis pathogens by PCR, and for HIV, syphilis and HSV-2 by serology. We used survey and STI test data to examine correlates of initial vs recurrent HSV and acute ulcer outcomes. Of men with HSV $(n=451)$, initial HSV was defined as a HSV-positive ulcer specimen and HSV-negative serology. Recurrent herpes was defined as a positive HSV specimen and serology. For all men, the acute ulcer outcome compared men who had initial HSV or who had Treponema pallidum, Haemophilus ducreyi, or Chlamydia trachomatis L1-L3 detected in their ulcer specimens (termed "acute ulcers") to men with GUD who did not have any of the four etiologies (termed "non-acute ulcers"). Correlates consisted of demographic characteristics and sexual behaviours often associated with STI.

Results Among all men, 29\% had an acute ulcer, and of those with HSV, 30\% had initial HSV. As compared to men with a non-acute ulcer, men with an acute ulcer were younger $(p<0.01)$, more likely to be single $(p<0.05)$, less likely to always use condoms with regular partners $(p=0.03)$, had more casual partners in the last 3 months $(p=0.03)$ but had fewer lifetime partners $(p=0.02)$. As compared to men with recurrent HSV, men with initial HSV were significantly more likely to be younger $(p<0.01)$, have multiple regular partners $(p=0.03)$, and report having sex in last week $(p=0.04)$, but were less likely to report ever exchanging sex for money $(p=0.01)$ and had fewer lifetime partners $(p<0.01)$.
Conclusions Findings suggest that young age and recent sexual behaviour were associated with initial HSV and acute ulcers in this group of men. These data emphasise the importance of targeting STI/HIV prevention programs in South Africa to young men engaging in high-risk sexual activities. To facilitate this, it is important to ensure that young men are equipped with the appropriate knowledge and skills to either avoid acquiring, or to seek early treatment for, STIs.

\section{P1-S5.11 HIGH PREVALENCE OF STIS AND RISK BEHAVIOURS AMONG PERSONS LIVING WITH HIV IN NICARAGUA: MISSED OPPORTUNITIES FOR PREVENTION}

doi:10.1136/sextrans-2011-050108.189

${ }^{1} \mathrm{~J}$ Goins, ${ }^{1} \mathrm{~L}$ M Romero, ${ }^{1} \mathrm{~F}$ de María Hernandez, ${ }^{1} \mathrm{~S}$ Delgado, ${ }^{1} \mathrm{~B}$ Alvarez, ${ }^{2} \mathrm{E}$ Beteta, ${ }^{3} \mathrm{G}$ Paz-Bailey, ${ }^{1} \mathrm{~S}$ Morales. 'Universidad del Valle, Guatemala City, Guatemala; ${ }^{2}$ Ministerio de Salud de Nicaragua, Managua, Nicaragua; ${ }^{3}$ Tephinet, Atlanta, USA

Background The UNAIDS Report on the Global AIDS Epidemic estimated that in 2009 Nicaragua had 6900 cases of HIV, almost double the 2001 estimate. Among people living with HIV in Nicaragua, it remains important to monitor sexually transmitted infections (STIs) and behavioural risk factors in order to inform STI prevention programs about the health needs of this population.

Methods In this cross sectional study, 200 people living with HIV were recruited consecutively from patients attending the Roberto Calderon Hospital in Managua, Nicaragua. Data was collected using computer-assisted self-interviews. Both men and women were tested for active syphilis (RPR titer $\geq 1: 8$ ) and herpes simplex virus type 2 (HSV-2) by serology, and for Chlamydia trachomatis, Neisseria gonorrhoeae, Trichomonas vaginalis, and Mycoplasma genitalium, by PCR. Acute STI was defined as infection with active syphilis or any of the PCR diagnosed STIs. We examined associations between acute STI infection and reported condom use as well as between STI infection and gender using simple logistic regression.

Results The most prevalent STI among study participants was HSV-2 (81.5\%). Active syphilis was diagnosed in $6.0 \%$ of the population. Men were less likely to be infected with an acute STI than women $(\mathrm{OR}=0.24 ; 95 \%$ CI 0.10 to 0.57 , Abstract P1-S5.11 table 1). A high percentage of participants reported having an HIV positive stable partner at the time of the interview (85.1\%). Consistent condom use in the last year was reported to be $59.9 \%$ with stable partners, $66.7 \%$ with commercial sex partners and $74.6 \%$ with occasional partners. Condom use at last sex was found to be protective for infection with acute STI $(\mathrm{OR}=0.38$; $95 \% \mathrm{CI} 0.17$ to 0.85).

Abstract P1-S5.11 Table 1 Prevalence of STls among persons living with HIV and OR by gender

\begin{tabular}{|c|c|c|c|c|c|c|c|c|c|c|}
\hline & \multicolumn{3}{|c|}{ Total } & \multicolumn{3}{|l|}{ Men } & \multicolumn{3}{|c|}{ Women } & \multirow[b]{2}{*}{$O R^{*}(95 \% \mathrm{CI})$} \\
\hline & $\mathbf{N}$ & $\mathbf{n}$ & $\%(95 \% \mathrm{Cl})$ & $\mathbf{N}$ & $\mathbf{n}$ & $\%(95 \% \mathrm{CI})$ & $\mathbf{N}$ & n & $\%(95 \% \mathrm{CI})$ & \\
\hline HSV-2 & 200 & 163 & 81.5 (75.4 to 86.6$)$ & 107 & 84 & 78.5 (69.5 to 85.9$)$ & 93 & 79 & 85.0 (76.0 to 91.5$)$ & $0.65(0.31$ to 1.35$)$ \\
\hline Syphilis & 199 & 23 & $11.6(7.5$ to 16.8$)$ & 106 & 13 & $12.3(6.7$ to 20.1$)$ & 93 & 10 & 10.8 (5.3 to 18.9$)$ & $1.16(0.48$ to 2.79$)$ \\
\hline Active syphilis & 199 & 12 & $6.0(3.2$ to 10.3$)$ & 106 & 6 & 5.7 (2.1 to 11.9$)$ & 93 & 6 & $6.5(2.4$ to 13.5$)$ & 0.87 (0.27 to 2.80$)$ \\
\hline Bacterial vaginosis & 81 & 23 & $28.4(18.9$ to 39.5$)$ & - & - & - & 81 & 23 & $28.4(18.9$ to 39.5$)$ & - \\
\hline Chlamydia trachomatis & 198 & 4 & $2.0(0.6$ to 5.1$)$ & 107 & 1 & $0.9(0.0$ to 5.1$)$ & 91 & 3 & $3.3(0.7$ to 9.3$)$ & $0.28(0.03$ to 2.71$)$ \\
\hline Neisseria gonorrhoeae & 198 & 0 & $0(0.0$ to 1.8$) \dagger$ & 107 & 0 & $0(0.0$ to 3.4$) \dagger$ & 91 & 0 & $0(0.0$ to 4.0$) \dagger$ & - \\
\hline Mycoplasma gentitalium & 198 & 9 & $4.6(2.1$ to 8.5$)$ & 107 & 1 & $0.9(0.0$ to 5.1$)$ & 91 & 8 & 8.8 (3.9 to 16.6$)$ & $0.10 \neq(0.01$ to 0.80$)$ \\
\hline Trichomonas vaginalis & 198 & 12 & 6.1 (3.2 to 10.6$)$ & 107 & 0 & $0(0.0$ to 3.4$) \dagger$ & 91 & 12 & 13.19 (7.0 to 21.9$)$ & - \\
\hline Any acute STI & 197 & 31 & 15.7 (10.9 to 21.6$)$ & 106 & 8 & $7.5(3.3$ to 14.3$)$ & 91 & 23 & 25.3 (16.7 to 35.5$)$ & $0.24 \S(0.10$ to 0.57$)$ \\
\hline
\end{tabular}

${ }^{*}$ Referent gender category: Women

†One-sided, $97.5 \% \mathrm{Cl}$.

$\neq \mathrm{p}$ value $<0.05$.

sp value $=0.001$

\Active syphilis, Chlamydia trachomatis, Neisseria gonorrhoeae, Mycoplasma gentitalium or Trichomonas vaginalis. 
Conclusion Despite personal experience with HIV, risky sexual behaviours and STI prevalence remain a problem in persons living with HIV in the capital city of Nicaragua. In order to prevent reinfection between serodiscordant partners as well as continued STI transmission, it is important for STI prevention programs to continue to develop their sexual health services, focusing on improved condom access and promotion.

\section{P1-S5.12 POPULATION ATTRIBUTABLE RISK FOR CHLAMYDIA INFECTION IN A COHORT OF YOUNG INTERNATIONAL TRAVELLERS (BACKPACKERS) AND RESIDENTS IN AUSTRALIA}

doi:10.1136/sextrans-2011-050108.190

H Wand. University of New South Wales, Sydney, Australia

Aim To estimate the population attributable risk (PAR) for Chlamydia trachomatis infection in young men and women in Sydney, Australia.

Method Multivariate logistic regression was used to examine the association between demographic, sexual behaviour and other potential risk factors on chlamydia positivity in young ( $\leq 30$ years) heterosexual international travellers (backpackers) and Australian residents attending a sexual health clinic. Point and interval estimates of PAR were calculated to quantify the proportion of chlamydia infections that can theoretically be prevented if a combination of risk factors is eliminated from a target population.

Results In males, the PAR associated with inconsistent condom use in the past 3 months was $65 \%$ (95\% CI 56 to 71\%) in backpackers compared to $50 \%$ (95\% CI 41 to $56 \%$ ) in non-backpackers and the PAR associated with reporting three or more female sexual partners in the past 3 months was similar between male backpackers and non-backpackers; 33\% (95\% CI 28 to 40\%) and 36\% (95\% CI 32 to $41 \%$ ), respectively. In females, the PAR associated with inconsistent condom use in the past 3 months was 51\% (95\% CI 42 to $59 \%$ ) in backpackers compared to $41 \%$ ( $95 \%$ CI 31 to $51 \%$ ) in non-backpackers, and the PAR associated with reporting three or more male sexual partners in the past 3 months was 14\% (95\% CI 11 to $18 \%$ ) in backpackers compared to $30 \%$ (95\% CI 25 to $37 \%$ ) in non-backpackers.

Conclusion These findings suggest that the largest number of chlamydia infections could be avoided by increasing condom use, particularly in backpackers. Reporting multiple partners was also associated with a large proportion of infections and the risk associated with this behaviour should be included in health promotion strategies.

\section{P1-S5.13 RECREATIONAL DRUG USE DURING SEX IS ASSOCIATED WITH STI AMONG CLIENTS OF A LARGE STI OUTPATIENT CLINIC IN AMSTERDAM, THE NETHERLANDS}

doi:10.1136/sextrans-2011-050108.191

\begin{abstract}
${ }^{1} \mathrm{M}$ Heiligenberg, ${ }^{1} \mathrm{P}$ Wermeling, ${ }^{1} \mathrm{M}$ van Rooijen, ${ }^{1} \mathrm{~A}$ Urbanus, ${ }^{1} \mathrm{M}$ Prins, ${ }^{2} \mathrm{R}$ Coutinho, ${ }^{1}$ M Schim van der Loeff. ${ }^{1}$ Public Health Service Amsterdam, Amsterdam, Netherlands; ${ }^{2}$ National Institute for Public Health and the Environment, Bilthoven, Netherlands
\end{abstract}

Background Previous studies have shown that recreational drug use is associated with high-risk sexual behaviour and STI. Little is known about the relationship between the use of specific recreational drugs during sex, sexual behaviour and the risk for any STI (chlamydia (CT), gonorrhoea (NG) and/or syphilis) or the three
STI separately in heterosexual men, men having sex with men (MSM) and women. We assessed the prevalence of recreational drug use during sex, and assocations of drug use during sex with high-risk sexual behaviour and STI.

Methods Attendees of the STI clinic of the Public Health Service of Amsterdam were interviewed during three waves of a bi-annual anonymous survey (2008-2009) about sexual behaviour in the preceding 6 months (number of steady and casual sex partners, condom use during vaginal or anal sex) and specific recreational drug use just before or during sex in the preceding 6 months (cannabis, XTC, poppers, heroine, cocaine, GHB, apomorfine, amphetamine, methylamphetamine, mushrooms, ketamine and explosion). Participants were tested for CT, NG and syphilis. Associations between drug use during sex and high-risk sexual behaviour and STI were analysed using multivariable logistic regression analyses. Analyses were done separately for heterosexual men, men who have sex with men (MSM) and women.

Results In total, 1012 heterosexual men, 749 MSM and 1254 women participated in this study. Of these, $11.7 \%$ had CT, 3.5\% NG and $1.4 \%$ syphilis. Recreational drug use during sex in the previous 6 months was reported by $22.5 \%$ of heterosexual men, $52.7 \%$ of MSM and $15.6 \%$ of women. In all three groups, drug use during sex was associated with unprotected vaginal and anal sex and more casual sex partners. In multivariable analyses among MSM, adjusting for age, ethnicity, educational level and sexual behaviour, poppers use was associated with any STI (adjOR 1.8, 95\% CI 1.2 to 2.6) and with NG (adjOR 2.6, 95\% CI 1.6 to 4.2), and GHB use with syphilis (adjOR 2.3, 95\% CI 1.0 to 5.0 ). In multivariable analyses among women, GHB use was associated with any STI (adjOR 4.7, 95\% CI 1.8 to 12.2 ) and CT (adjOR 3.5, 95\% CI 1.1 to 11.4). In heterosexual men, drug use during sex was not associated with STI.

Conclusions STI clinic clients frequently report recreational drug use during sex and this is associated with high-risk sexual behaviour in heterosexual men, MSM and women. The use of drugs during sex is associated with STI in MSM and women, but not in heterosexual men.

\section{P1-S5.14 RISK FACTORS ASSOCIATED WITH HIV ACOUISITION: A COMPARATIVE ANALYSIS OF OLDER AND YOUNGER WOMEN WHO PARTICIPATED IN THE MDP301 TRIAL IN JOHANNESBURG}

doi:10.1136/sextrans-2011-050108.192

${ }^{1} \mathrm{~S}$ Delany-Moretlwe, ${ }^{1} \mathrm{~A}$ Nanoo, ${ }^{2} \mathrm{~A}$ Nagpal, ${ }^{1} \mathrm{H}$ Moultrie, ${ }^{1} \mathrm{~S}$ Delany, ${ }^{1} \mathrm{H}$ Rees. ${ }^{1} \mathrm{WHI}$, Hillbrow, South Africa; ${ }^{2}$ LSHTM, UK

Background Young women are known to be particularly at risk for HIV infection. Identifying characteristics associated with HIV-acquisition in younger and older women could assist with developing effective interventions which target the determinants of HIV.

Methods The MDP301 study was an international, multi-centre, randomised controlled trial to assess the efficacy of $0.5 \%$ PRO2000/ 05 microbicide gel. 2508 HIV-negative women, $\geq 18$ years, were enrolled in Soweto and Orange Farm and followed up for 12 months. Associations between baseline demographic, behavioural and clinical risk factors and HIV acquisition were assessed using univariate Poisson regression.

Results Data on 2451 women were analysed. 110 seroconversions were observed over 2356.5 woman-years (wy). Overall, HIV incidence was $46.7 / 1000$ wy (95\% CI 38.7 to 56.3). Younger women (18-24 years) were more likely to acquire HIV (IRR1.4, 95\% CI 1.0 to 2.0) than older women ( $\geq 25$ years). Difficulty accessing money for medical treatment (IRR $1.5,95 \%$ CI 0.06 to $2.0, p=0.019$ ) 